Basic Health Sciences

Poster

Abstract ID: 131

\title{
The Role of Training for Dental Students in Scoring Caries
}

\author{
Zurainie Abllah
}

Kulliyyah of Dentistry, International Islamic University Malaysia

Introduction: Training of dental students is an essential part of the teaching- learning activities in order to obtain reliable results in scoring of dental caries. The objectives of this study are to examine consistency in scoring of caries over a 3-month period following baseline training, calibration and retraining. Methods: Three examiners (dental students) received baseline training/calibration by a "gold standard" examiner (lecturer). This was followed by a re-calibration and retraining 3 months later. A set of 20 extracted teeth was used for the baseline training and for the re-calibration purposes. The International Caries Detection and Assessment System (ICDAS) was used to classify the visual caries lesion severity (0-6 scale) of surface of permanent teeth. The examination utilized a clinical light, dental mirror, dental probe and air syringe. Kappa (unweighted: Kappa) statistics were used to determine inter-examiner reliability at baseline and re-calibration. Results: For caries score criteria, the baseline calibration on 20 specimens indicated an inter-rater kappa ranging from 0.37-0.69. Re-calibration on the same 20 specimens 3 months later indicated an inter-rater kappa of 0.79-0.89. Activity kappa was in the poor to good range. All examiners improved with time. Conclusions: Training contributes to the consistency and reliability of dental caries scoring among dental students.

KEYWORDS: training, ICDAS, caries, dental student 